Bio - grafía. Escritos sobre la Biología y su Enseñanza. ISSN 2027-1034

Edición Extraordinaria. p.p. 441 - 447

Memorias del IX Encuentro Nacional de Experiencias en Enseñanza de la Biología y la Educación Ambiental. IV Congreso Nacional de Investigación en Enseñanza de la Biología.

\title{
EL CAMINO DE LA NORMAL AL PRIMER DÍA DE ESCUELA
}

\begin{abstract}
"Me acuerdo entrar ese día a la Iglesia entrar por toda la mitad, como entran los novios y a cada una nos dieron nuestra plaquita, cada uno "profesora" y así decía mi placa "profesora Micaela del Pilar Montenegro Aldana", ¿ir a vender libros? !no;. !Yo necesito de los niños; necesito de sus risas, de sus miradas... ellos son parte de mi vida"
\end{abstract}

\section{Micaela Montenegro Adana ${ }^{1}$ \\ Rebeca Urazán Benitez ${ }^{2}$ Nixon Medina Talero.}

\section{RESUMEN}

Son las historias del día a día las que constituyen la escuela. La voz de cada uno de nuestros maestros, sobre todo aquellos con más experiencia, posee un profundo caudal de experiencias silenciadas que merecen ser recuperadas y reconocidas. Precisamente, La investigación narrativa (Narrative inquiry), se ha preocupado por retornar a la raíz y el corazón de la enseñanza, nuestros profesores.

El relato, que se encuentra a continuación, surge de una investigación (Narrative inquiry), en la que se realizaron una serie de entrevistas a cinco profesores con más de 20 años de experiencia; Buscamos recopilar relatos que nos permitieran comprender la relación de su formación (normal, pregrado y posgrado) en relación con su propia práctica profesional. Un relato, para ser reconocido como tal, debe poseer una estructura básica alrededor de un problema (un detonante narrativo). En el caso de esta bio-crónica, Micaela nos cuenta una parte de su historia; esta maestra, con más de 24 años de experiencia, nos narra el camino vivido desde la normal hasta su primer día de clase como profesora. Esta historia que podría ser tan natural, dibuja unos bellísimos momentos, cuando la maestra se ve enfrentada a toda clase de obstáculos, por su origen humilde y sus escasos recursos económicos, para obtener el sueño de su vida, trabajar en el mejor colegio de Bogotá.

La siguiente crónica se encuentra dividida en varios momentos, jerarquizados con subtítulos, que nos permiten hilar aquellos momentos trascendentales. Tratamos de retirar

\footnotetext{
${ }^{1}$ Docente I.E.D Francisco Antonio Zea. Especialista en orientación educativa y desarrollo humano. Docente Ciencias Naturales y Educación Ambiental -Primaria.

2 Docente Colegio La Concepción C.E.D. Magister (C) en Educación. Contacto: rebelepidoptera@gmail.com. Docente Ciencias Naturales y Educación Ambiental.

3 Docente Institución Educativa Rural El Destino. Estudiante doctorado en Educación. Contacto: nixonmedita@gmail.com. Docente Ciencias Naturales y Educación Ambiental.
} 
Bio - grafía. Escritos sobre la Biología y su Enseñanza. ISSN 2027-1034

Edición Extraordinaria. p.p. 441 - 447

Memorias del IX Encuentro Nacional de Experiencias en Enseñanza de la Biología y la Educación Ambiental. IV Congreso Nacional de Investigación en Enseñanza de la Biología.

nuestra propia voz como investigadores, para dar un protagonismo absoluto a la historia de la profesora, respetando en todo momento la integridad del relato. Nos repensamos la investigación, considerando que en muchos momentos es necesario que las narraciones hablen por sí solas y muestren su propio esplendor.

PALABRAS CLAVE: Investigación narrativa, Relato, crónica, Historias de vida, experiencia.

\section{ABSTRACT:}

It is the stories of the day to day that make up the school. The voice of each one of our teachers, especially those with more experience, has a deep flow of silenced experiences that deserve to be recovered and recognized. Precisely, Narrative inquiry, has been concerned to return to the root and the heart of teaching, our teachers.

The following story emerges from a research (Narrative inquiry), in which a series of interviews were conducted with five professors with more than 20 years of experience; We sought to compile stories that allowed us to understand the relationship of their training (normal, undergraduate and postgraduate) in relation to their own professional practice. An account, to be recognized as such, must have a basic structure around a problem (a narrative detonator). In the case of this bio-chronicle, Micaela tells us a part of its history; This teacher, with more than 24 years of experience, tells us the way she lived from the normal until her first day of school as a teacher. This story that could be so natural, draws beautiful moments, when the teacher is faced with all kinds of obstacles, humble origin and scarce economic resources, to obtain the dream of his life, to work in the best school in Bogota .

The following chronicle is divided in several moments, hierarchical with subheadings, that allow us to spin those transcendental moments. We try to withdraw our own voice as researchers, to give an absolute protagonist to the history of the teacher, respecting at all times the integrity of the story. We rethink the investigation, considering that in many moments it is necessary that the narrations speak for themselves and show their own splendor.

KEYWORDS: Narrative investigation, Story, chronic, Life stories, experience.

\section{LA NORMAL - LOS SUEÑOS DE UNA NIÑA-}

Mi Normal transcurrió desde sexto hasta once y las experiencias fueron múltiples. Recuerdo que en la Normal yo me paraba en frente de mis compañeras y les decía: Bueno, voy a suponer que yo estoy en un colegio [me paraba allá toda estirada y les decía] vamos a suponer que yo estoy trabajando en la ciudad, porque yo me voy a ir para 
Bio - grafía. Escritos sobre la Biología y su Enseñanza. ISSN 2027-1034

Edición Extraordinaria. p.p. 441 - 447

Memorias del IX Encuentro Nacional de Experiencias en Enseñanza de la Biología y la

Educación Ambiental. IV Congreso Nacional de Investigación en Enseñanza de la Biología.

la ciudad-, y todas me decían: -jajaja, si eso es difícil, ¿con qué plata?-. Todas éramos provincianas, todo el mundo de pueblos: Jubalá, Gachetá, Une, Manta, Ubateque, Tibirita, Macheta... todos esos pueblos de la región del Valle de Tenza y de la región del Guavio.

-!Jajaja, Micaela sí sueña!- me decían y les dije: -Me voy para la ciudad... lo verán, Micaela Montenegro llegando a la ciudad, y voy a trabajar en uno de los mejores colegios, yo les decía eso. Entonces yo me paraba allá y yo les decía: -bueno; vamos a tratar tal tema, pregunten, yo les voy a dictar la clase- y empezábamos así... Esos tiempos. Cosa tal, que terminé en 1985, el 5 diciembre fue mi grado en la iglesia del pueblo, el grado de las Normalistas. Luego de salir de la Normal (en 1985), me fui para la finca y duré 1986, 1987 y mitad de 1988, cortando caña y cogiendo café.

Sé todo el proceso del café y a veces en ciencias, cuando vemos lo de las plantas, yo lo explico, porque me encanta. Además de todo el proceso del café lo sé hacer, me sé organizar con obreros porque yo me compraba mis lotes de café y los negociaba, siempre me ha gustado vender. Compraba mi lote grande de café y lo negociaba con el dueño vale tanto-, decía, yo lo pesaba y listo. Yo calculaba, bueno una, dos cargas saco acá... vale tanto y eso fue en esa época. También con la caña de azúcar, me compraba mi lote y lo llevaba para la molienda, para la miel y obviamente la miel la vendía para todo el proceso de la panela. Compraba mi leche, hacía mis quesos y vendía mis quesos ¿para qué?... ¡Para venirme a Bogotá!, porque Bogotá era la gran ciudad.

Manta es mi pueblo, donde nací y crecí. Gacheta, pegadito, donde todavía funciona mi Escuela Normal Superior. Hacia mis quesos, vendía mis quesos, los llevaba a vender. Dos años y medio duré trabajando duro en la finca ahorrando para venir a la gran ciudad que es Bogotá a estudiar, porque mi meta era seguir, no quedarme en el campo porque entonces ¿para qué estudié? Y haciendo mis trabajos con lo del café, con lo de los quesos y con el proceso de la caña de azúcar, ahorré para comprar ropa y para mis semestres, para venir a la ciudad.

\section{LA LLEGADA A BOGOTÁ}

Viajé en 1988, pero finalmente entre a estudiar hasta el 1993; pero nunca me gasté el dinero, el dinero siempre lo tuve ahí. El temor de entrar a estudiar, pues pensaba "esa plata es sagrada", porque la había ganado con el sudor de la frente y no sólo de la frente, eso sería mentira, eso no sólo suda la frente, es el sudor de todo el cuerpo.

Viajé para Bogotá en julio del 1988, me fui a vivir con Ignacio, mi hermano. Viajábamos a Tunja, yo entraba a los colegios con propiedad y yo cogía los libros de mi hermano, mi hermano empezó con unos libros llamados: Me Preparo para ganar primero de primaria, Me preparo para ganar segundo, tercero, cuarto, quinto y así sucesivamente. Esos fueron los primeros libros de mi hermano. Luego libros para ingresar al bachillerato, para el ICFES. 
Bio - grafía. Escritos sobre la Biología y su Enseñanza. ISSN 2027-1034

Edición Extraordinaria. p.p. 441 - 447

Memorias del IX Encuentro Nacional de Experiencias en Enseñanza de la Biología y la Educación Ambiental. IV Congreso Nacional de Investigación en Enseñanza de la Biología.

Él ha escrito bastante (Ignacio, mi hermano), mejor dicho, no recuerdo ahorita, tiene uno también del 1290 que es reciente, esos los encuentra en la Cooperativa del Magisterio. Entonces con esos libritos que eran verde con amarillo, jamás se me olvidará, tenían una franja verde y una franja amarilla y nos íbamos para Tunja en un carrito pequeñito porque los recursos no daban para un carro lujoso, grande. Un carrito pequeñito y ahí empacábamos los libros y eso sí, tocaba irse bien arreglado, porque de lo contrario graves. Y llegar, y a mí no me daba miedo... Entrábamos y mi hermano hablaba con el rector o con la rectora, con quien fuera hablaba y yo bueno, me decía -pase allá Micaela-, yo iba y le hablaba a los profesores, jeso me compraban! Vendí, vendí bastante.

\section{YO NECESITO SER PROFESORA}

Mi hermano me insistía mucho en que escribiera, yo me fui a vivir con él y me dijo siéntese y escriba-. Yo escribía un poquito y la verdad ¡Que pereza! Yo le decía a mi hermano -eso lgnacio, mejor ayúdenme a conseguir mi colegio, que yo quiero trabajar con los niños, a mí me hace falta la Normal, me hacen falta los muchachos de las escuelas de la Normal, ayúdeme más bien que yo quiero entrar a trabajar-.

Y me puse recordar todo lo que viví en la Normal y todo lo que nos decían nuestras profesoras, la de fundamentos, la de metodología, la de práctica, me acuerdo entrar ese día a la Iglesia entrar por toda la mitad, como entran los novios y a cada una nos dieron nuestra plaquita, cada uno "profesora" y así decía mi placa "profesora Micaela del Pilar Montenegro Aldana", ¿ir a vender libros? !no!. Yo necesito de los niños, necesito de sus risas, de sus miradas, necesito... ellos son parte de mi vida.

La familia siempre tuvo un amigo, él trabajó muchos años en esta localidad, un rector, se llamaba Hernán Montaño. Él murió de un cáncer terrible, muy amigo de la familia. Y un buen día llamó:

- ¿lgnacio? -, me pregunto. -no, no señor, no está-, le dije

-Doctor Montaño y ¿cómo me le va? -

-! holai, Pilarcita, usted es la menor- me dijo. - ¿Cómo está?” - le dije: -Yo, pues bien-

¿Ignacio? - pregunto. -No, no señor, él está trabajando- respondí.

- ¿Y tú que andas haciendo?"- Me pregunto.

-No doctor, imagínense que sin empleo, yo estoy aquí con Ignacio, pero me siento sin empleo porque yo quiero trabajar es en un colegio- le dije

- ¡Tengo palanca Montenegroi, iTengo palanca!, Tráigame mañana estos y estos papeles, te voy a ubicar- me dijo.

Y efectivamente, ese señor está en el cielo porque ese señor ayudó, me ayudó y ayudó a muchísima gente, nos dio el pan en la mesa, nos dio la felicidad de poder... a mí me dio la 
Bio - grafía. Escritos sobre la Biología y su Enseñanza. ISSN 2027-1034

Edición Extraordinaria. p.p. 441 - 447

Memorias del IX Encuentro Nacional de Experiencias en Enseñanza de la Biología y la

Educación Ambiental. IV Congreso Nacional de Investigación en Enseñanza de la Biología.

felicidad de poder trabajar con los niños, porque quizás si yo no hubiera encontrado rápido mi colegio que es este, la escuela de primaria. Yo me hubiese ido por otro lado, porque no me iba a devolver... No, no, porque yo siempre digo lo que uno quiere toca lucharlo. Yo dije devolverme al campo no, yo le di dos años y medio duro al trabajo en el campo, pero devolverme ni por el chiras, yo necesito trabajar y quiero trabajar es con los niños.

\section{-Así Me TOQUe EN BOTAS DOCTOR-}

Efectivamente al otro día fui le llevé papeles, eso fue en febrero del 1989, porque ya había pasado tiempo. Pasaron unos días y listo, me llamó y me dijo: -Pilar, tiene empleo en Usme, en una escuelita que queda en Usme, hace frío...- me contó -hace mucho frío, llueve bastante...-, le dije: -no interesa doctor eso así me toque con botas, en la marcha, pero para mí lo más importante es llegar a donde haya niños-.

\section{EL PRIMER díA DE CLASE}

El 27 marzo de 1989 el corazón me latía así (gestos de latido), iporque iba a hacer lo que yo había estudiado! y a la vez sentía nervios. Decía "¿cómo voy a dictar una clase?" y volvía y decía "ipero si yo estuve en la Normal cuatro años!, yo trabajaba allá prácticamente, no me pagaban, pero yo trabajaba dando mis clases, yo puedo".

Me recibió Danilo, un señor hosco, hoy en día lo quiero mucho, guerreamos mucho y aprendí mucho de él. Un señor hosco, -eso tiene tercero de primaria, es un curso indisciplinado-, me dijo, me parece escucharle la voz, bien puesto, con su voz firme.

Me dijo -usted es Montenegro, ¿usted es hermana de Juan Montenegro? - Le dije -sí, yo soy la hermana, si señor-, me dijo: -cómo le parece que Juan Montenegro es el director de la OEA y yo fui maestro de Juan, Juan me hizo la vida imposible...-. Pues pensé rápidamente, esto aquí va a haber guerra. Dije: - jah!, vea cómo son las cosas- y me respondió: -su hermano fue duro conmigo, porque su hermano es un rector estricto- y lo que le contesté, recuerdo -sabe qué director... (no es como en esta época coordinador sino director), le dije: - ¿sabe qué señor director?, Juan es Juan, mi nombre es Micaela Montenegro, somos dos personas diferentes, somos hermanos sí, pero somos distintos. Él allá y yo acá-, el tipo me miró como diciendo esta no es tan caída del zarzo.

-Trabaja con terceros Montenegro, jeste es indisciplinado!, hay un niño trae puñaleta, hay un niño que no sé qué-. Bueno, empezó con todos, y yo recuerde todo lo de la Normal. Yo parecía un libro, páginas y páginas atrás. Bueno, cómo actuar, cómo hacer, eso era como si fuera a hacer una obra de teatro, como si fuera a presentar una obra de teatro en mi primer día.

Siempre lo recuerdo. Entro a ese curso. Papel iba, papel venía, chifle uno... Entró Danilo, se paró en la puerta -Buenas tardes estudiantes, la profesora Montenegro viene a acompañarlos-. respiré -si me la dejo ganar...- pensé, porque ¿con quién hablaba?, no conocía a nadie... Pero, aun así, dentro de mí la emoción, era la emoción de ver que ya 


\title{
Bio - grafía. Escritos sobre la Biología y su Enseñanza. ISSN 2027-1034
}

\section{Edición Extraordinaria. p.p. $441-447$}

\author{
Memorias del IX Encuentro Nacional de Experiencias en Enseñanza de la Biología y la \\ Educación Ambiental. IV Congreso Nacional de Investigación en Enseñanza de la \\ Biología.
}

no tenía que coger a hacer otros trabajos sino iba a estar con los niños, iba a trabajar con ellos, eso pudo más que cualquier otra cosa.

Él se paró ahí y me dijo, como con el toro, como cuando sueltan el toro y queda uno en la mitad, sin ser torero. ¡Pero yo sí era torera, porque sí me había preparado, me había preparado para ser maestra! Entonces, él se paró ahí en la puerta, no le hacían caso, los chinos hablaban, se mandaban papeles: -la profesora... joven que se siente...- Les decía, al fin hicieron silencio -... los va a acompañar. Profesora la dejo con su curso-. Cerró la puerta y se fue, cierra la puerta y me deja con los niños.

¿Qué hice? Así aplaudiendo y llevando el ritmo con una canción de la Normal, empecé a cantarles, fui caminando; entonces me fui parando, me fui moviendo y los chicos se fueron parando, se fueron moviendo al ritmo de lo que yo cantaba, cantaba como un tarro, pero cantaba y los chicos cantaban conmigo. Es que lo que se hereda no se hurta y lo que uno aprende en la Normal, lo digo yo, lo que uno aprende en la Normal, lo que va aprendiendo en la Normal de uno al 100\%; quizás se le haya olvidado el $15 \%$, el resto lo tengo presente. De un momento a otro yo me empecé a mover y los chicos... eso yo iba en tacones, que en esa época los tacones eran largos, porque la emoción de que iba a usar tacón, y para ello me compré los más altos (pero yo ensayé en la casa y los manejaba). Los chicos se empezaron a mover, se acabó la canción, los chicos sigan con el papel y yo con otra canción.

\section{La Clase del Ciclo del Agua. - Terminé más cansada que CARgando caña... -}

Empecé a hacer mañita, fui abriendo la puerta, las dos puertas, que tiene el salón y fui cantando. Los chicos me preguntaron: -¿Profe por qué abre?-, les dije -está haciendo como calor-, -profe, pero si está lloviendo- me respondieron, les dije: -sí, pero yo tengo calor-. Bueno, porque yo decía es que ese encierro, estos chicos bien inquietos, este salón cerrado, lloviendo y ellos queriendo mirar. Empezó a caer granizo y los chicos friegue, empecé con mañitas a sacarlos, a sacarlos y los puse en la baranda y les di una clase maravillosa sobre los ciclos del agua.

Danilo apenas se paró allá: - ¿y ésta maestra qué está haciendo? -. Yo fui sin importa qué, no me importó mojarme y cogí el hielo, empecé a explicarles, precipitación, bueno todo lo del ciclo del agua, luego los pasé, cuaderno, saquemos hojitas, miremos... plastilina ¿alguno trajo plastilina? - pregunte, -Si profe, yo traje- respondían, - ¿crayolas?preguntaba, -Si profe- me decía un chico, - ¿temperas?, Tráigalas- . Los organicé en grupos, porque pensé, cuando puse mis pies ahí me dijo que era el curso más indisciplinado. -Si estos chicos me la ganan hoy, fracasé como maestro, porque los chicos me cogen el lado flaco- pensaba, ¿quién va a perder el año? pues Yo. Que unos trajeron crayolas, que otros plastilina, que los otros en el cuaderno, los organicé por grupos, dibujitos, escribir, luego les hice una síntesis, llegó el recreo. Entramos, ya fue la clase de religión y terminé.

Soy sincera, si yo no hubiese tenido las herramientas de la Normal ese día, yo hubiera dicho- no, hasta luego señor Danilo, que le vaya muy bien- y me hubiera ido a taconear a otro lado. Y así, terminé, pero terminé más cansada que cuando echaba en mi hombro la 
Bio - grafía. Escritos sobre la Biología y su Enseñanza. ISSN 2027-1034

Edición Extraordinaria. p.p. 441 - 447

Memorias del IX Encuentro Nacional de Experiencias en Enseñanza de la Biología y la Educación Ambiental. IV Congreso Nacional de Investigación en Enseñanza de la Biología.

caña, la caña que yo amarraba con un lacito en el hombro, porque no había burro, el burro era la espalda. Terminé más cansada que cargando caña, pero salí satisfecha, porque sentí, porque fue mi primer día, manejé mi grupo y esto es lo mío. Aquí estoy y aquí me quedo.

\section{AÑOS DE HISTORIA Y LA QUE SE SIGUE ESCRIBIENDO}

Cuando me nombraron, me nombraron en el 1993, tenía el dinero que traía de Manta, ahí quietico, eso nada de comprar zapatos, los tacones los compré con otra plata, esa plata era para estudiar... apenas me nombraron en 1993, en febrero, entonces el 20 julio me fui a estudiar mi licenciatura en pedagogía reeducativa.

Terminé en 1997 y en 1998 empecé para hacer la especialización en orientación educativa y desarrollo humano que terminé en el diciembre del 1999. ¡Y estoy en el mejor colegio!, como lo dije en la normal, -voy a estar en el mejor colegio de Bogotá- es el mejor porque estos niños son nobles, porque a estos niños uno les llega, yo siento que uno les llega, que molestan y que a veces es terrible, sí, hacen indisciplina, pero uno les llega. Este es el mejor colegio, para mí lo es, por eso no me he ido.

Ante la posibilidad de trasladarme cerca de mi lugar de residencia he dicho ¡no! A Usme llegué joven, amo mi Usme, amo mi comunidad de Usme, quiero irme cuando me pensione o si Dios me manda una enfermedad, porque es posible que sea y me pensionan por enfermedad quiero salir de Usme, o sino salir de Usme cuando cumpla mi ciclo de maestro.

Y aquí estoy, ya 24 años... y esta es parte de mi historia.

\section{BIBLIOGRAFÍA:}

- Bolivar, A. \& Domingo, J. (2006). La Investigación Biográfica y Narrativa en Iberoamérica: Campos de desarrollo y estado actual. Forum: Qualitative Social Research, 7 (4), 1-33.

- Bolívar, A. (2001). La investigación biográfico-narrativa en educación,. Enfoque y Metodología. Madrid, España: La Murralla.

- Bolívar, A., Domingo, J. \& Fernández, M. (2006). La Investigación BiograficoNarrativa en Educación: Enfoque y Metodología. Madrid, España: Editorial La Muralla.

- Connelly, F. M. \& Clandinin, D. J. (1995). Relatos de experiencia e investigación narrativa. En Larrosa, J. et al. (Eds), Déjame que te cuente. Ensayos sobre narrativa y educación. Barcelona, España: Laertes.

- De Certeau, M. (2000). La invención de lo cotidiano 1: Artes de Hacer. México: Universidad lberoamericana. 